\title{
Design and Performance of Nitride-based UV LEDs
}

\author{
Mary H. Crawford, Jung Han, Weng W. Chow, Michael A. Banas, Jeffrey J. Figiel, Lei Zhang and \\ Randy J. Shul \\ Sandia National Laboratories, Albuquerque, NM 87185-0601
}

\begin{abstract}
In this paper, we overview several of the critical materials growth, design and performance issues for nitride-based UV $(<400 \mathrm{~nm})$ LEDs. The critical issue of optical efficiency is presented through temperature-dependent photoluminescence studies of various UV active regions. These studies demonstrate enhanced optical efficiencies for active regions with Incontaining alloys ( InGaN, AlInGaN). We discuss the trade-off between the challenging growth of high Al containing alloys (AlGaN, AlGaInN), and the need for sufficient carrier confinement in UV heterostructures. Carrier leakage for various composition AlGaN barriers is examined through a calculation of the total unconfined carrier density in the quantum well system. We compare the performance of two distinct UV LED structures: GaN/AIGaN quantum well LEDs for $\lambda<360 \mathrm{~nm}$ emission, and InGaN/AlGaInN quantum well LEDs for $370 \mathrm{~nm}<\lambda<390 \mathrm{~nm}$ emission.

\section{INTRODUCTION}
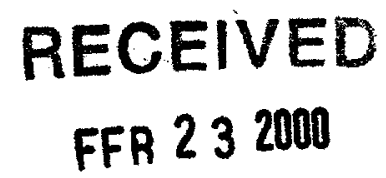

C.STI

While much of the research in the nitride field has focused on the development of blue and green LEDs and laser diodes, UV $(\lambda<400 \mathrm{~nm})$ LEDs based on GaN, AlGaN and/or AlGaInN active regions are also of great interest and are well suited to a number of applications. One of the most promising uses of a UV LED is as a high energy excitation source. In the biomedical and chemical sensing fields, UV LEDs can be used as compact and robust excitation sources of fluorescence. UV LEDs may also prove to be superior to blue LEDs in exciting phosphors for white lighting applications, due to improved color rendering. To date, there have been relatively few reports of UV LEDs based on the wide-bandgap nitride semiconductors. In particular, Akasaki et. al. [1] reported on a GaN/AlGaN double heterostructure (DH) LED with emission at $370 \mathrm{~nm}$ and up to $1.5 \%$ external quantum efficiency. Mukai, et. al. [2] have achieved an impressive $5 \mathrm{~mW}$ output at 371 $\mathrm{nm}$ from an InGaN/AIGaN DH LED with very low levels of indium (In) in the active region. That LED was reported to have up to 7.5\% external quantum efficiency but the efficiency dropped by more than an order of magnitude as the emission wavelength shifted to $368 \mathrm{~nm}$ with the total elimination of In from the active region. Shorter wavelengths have been achieved by Han, et. al. [3] through a GaN/AlGaN multiquantum well (MQW) structure. These LEDs demonstrated a 354 $\mathrm{nm}$ emission peak with a narrow FWHM linewidth of $5.8 \mathrm{~nm}$ and relatively low output powers of $12 \mu \mathrm{W}$ at $20 \mathrm{~mA}$. Thus, while one group has demonstrated high ( $>5 \%$ ) efficiency LED performance for $\lambda>370 \mathrm{~nm}$, many of the challenges inherent to the shorter wavelength emission regime still remain.

In this paper, we discuss a number of materials growth and design challenges for achieving high performance UV LEDS and present the performance of two distinct UV LED structures. In section 2, we describe the materials growth and the general heterostructure designs for the UV LEDs that we have developed. In section 3 we review critical materials and design issues such as optical efficiency of UV active regions, internal absorption effects, critical thickness limitations for AlGaN layers and carrier leakage. We report on the performance of GaN/AIGaN MQW LEDs with emission wavelengths $<360 \mathrm{~nm}$ as well as the performance of InGaN/AlInGaN MQW LEDs for $370 \mathrm{~nm}<\lambda<390 \mathrm{~nm}$ emission in sections 4 and 5 , respectively.
\end{abstract}

\section{MATERIALS GROWTH AND LED HETEROSTRUCTURE DESIGNS}

$\therefore$

The nitride materials described in this paper were grown in a high speed $(\sim 1200 \mathrm{rpm})$ rotating disk MOCVD reactor on two inch sapphire substrates. Different growth conditions were employed for non-indium containing alloys (GaN, AlGaN) as compared to indium containing alloys (InGaN, AlInGaN). The GaN and AlGaN growths [3] were typically carried out at $1000-1080^{\circ} \mathrm{C}$ with hydrogen as the carrier gas. Ammonia $\left(\mathrm{NH}_{3}\right)$, Trimethylgallium (TMGa), Trimethylaluminum (TMAl) were used as the N, Ga, and Al precursors, respectively. The growth of InGaN and AlInGaN [4] was carried out at 750$800^{\circ} \mathrm{C}$, with nitrogen as the carrier gas and Triethylaluminum (TEA) and Trimethylindium (TMI) as the Al and In sources, 
respectively. A standard two-step growth (550 and $1050{ }^{\circ} \mathrm{C}$ for the low and high temperatures, respectively) with a GaN low-temperature buffer layer $(-250 \AA)$ was used in this work.

We will focus on two general heterostructures for UV LEDs. The overall layer sequence is quite similar for the two structures, and is shown in Figure 1. The first design is an MQW LED structure with GaN quantum wells and $\mathrm{Al}_{\mathrm{x}} \mathrm{Ga}_{1-x} \mathrm{~N}$ barriers and emission in the $\lambda<360 \mathrm{~nm}$ region. The $\mathrm{n}$-GaN buffer layer is typically $3 \mu \mathrm{m}$ in thickness and Si-doped to a level of $2-5 \times 10^{18} \mathrm{~cm}^{-3}$. The $\mathrm{n}-\mathrm{AlGaN}$ cladding with $\mathrm{x}=0.15-0.20$ is approximately $400 \AA$ thick and doped to a similar level as the n-GaN layer. The multiquantum well region consists of 5 periods of $30 \AA$ thick GaN quantum wells and $70 \AA$ thick AlGaN ( $x=0.15-0.2)$ barriers. The p-AlGaN cladding is typically $400 \AA$ thick and the contact layer is $0.05-0.1 \mu \mathrm{m}$ thick pGaN. The second type of UV LED structure utilizes InGaN QWs and AlInGaN barriers in the MQW active region. The MQW region consists of $47 \AA$ thick $\operatorname{In}_{x} \mathrm{Ga}_{1-x} \mathrm{~N}$ quantum wells with $\mathrm{x}=0.04$ and $48 \AA$ thick $\mathrm{Al}_{\mathrm{y}} \mathrm{In}_{\mathrm{x}} \mathrm{Ga}_{1-\mathrm{x}-\mathrm{y}} \mathrm{N}$ barriers with $\mathrm{x}=0.04$ and $y=0.14$. The $p-G a N$ cap layer is thicker for these structures, due to the fact that the longer wavelengths emitted from the active regions are not as strongly absorbed by the $\mathrm{p}$-GaN layer. Thicknesses of $0.1 \mu \mathrm{m}$ to $0.25 \mu \mathrm{m}$ have been used.

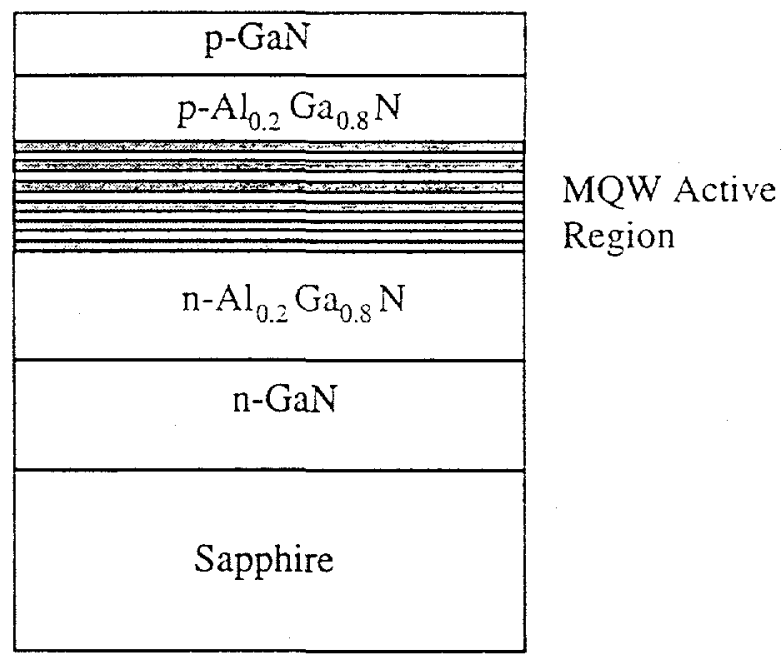

Figure 1: Schematic of UV LED MQW heterostructures

\section{GROWTH AND DESIGN CHALLENGES FOR HIGH EFFICIENCY UV LEDS}

The performance of nitride-based UV LEDs has yet to reach that of commercially available blue and green LEDs. Although relatively good performance has been reported in the near UV region of $\lambda>370 \mathrm{~nm}$, there exist a number of distinct challenges for achieving high efficiency at the UV wavelengths, and these challenges become particularly difficult for wavelengths shorter than $370 \mathrm{~nm}$. In this section, we review specific challenges that limit UV LED performance, including optical efficiency of the active regions, internal absorption effects, critical thickness limitations and carrier leakage.

\subsection{Optical Efficiency of UV LEDs}

One of the most intriguing issues in the nitride field concerns the high optical efficiency of nitride-based blue and green LEDs. Specifically, although a great deal of progress has been made in the development of InGaN-based light emitters, the role played by indium (In) in contributing to the optical efficiency is still quite controversial. A number of groups have proposed that the inhomogeneity of In incorporation results in carrier localization at In-rich regions and that this localization leads to enhanced optical efficiency [6-8]. Support of this hypothesis is found in cathodoluminescence experiments that demonstrate a variation of the PL emission energy on the microscale, suggesting that In composition variations on the order of several percent are possible [9]. Further insight is gained by the time-resolved spectroscopy experiments of InGaN quantum well structures performed by Narukawa, et. al. [10], which suggest that the density of non-radiative centers and possibly the non-radiative recombination mechanism itself is altered when In is included in the growth. A similar result was 


\section{DISCLAIMER}

This report was prepared as an account of work sponsored by an agency of the United States Government. Neither the United States Government nor any agency thereof, nor any of their employees, make any warranty, express or implied, or assumes any legal liability or responsibility for the accuracy, completeness, or usefulness of any information, apparatus, product, or process disclosed, or represents that its use would not infringe privately owned rights. Reference herein to any specific commercial product, process, or service by trade name, trademark, manufacturer, or otherwise does not necessarily constitute or imply its endorsement, recommendation, or favoring by the United States Government or any agency thereof. The views and opinions of authors expressed herein do not necessarily state or reflect those of the United States Government or any agency thereof. 


\section{DISCLAIMER}

Portions of this document may be illegible in electronic image products. Images are produced from the best available original document. 
obtained by Kumano, et. al. [11] who suggest that increased optical efficiency is due to reduced non-radiative recombination centers with In incorporation. Thus, it is clear that a strong consensus has not emerged as to how the presence of In in the QWs affects the optical efficiency and whether it is absolutely necessary for achieving high efficiency nitride LEDs.

This issue of whether In-containing QWs are needed for high efficiency is especially critical if we look at UV LED structures. Clearly the GaN MQW LEDs do not contain In in the active regions, and therefore would not benefit from the proposed improvements in optical efficiency seen in the blue and green LEDs. Furthermore, in order to achieve LED emission at $\lambda<390 \mathrm{~nm}$ from InGaN. MQW structures, the In composition must be reduced to relatively small values $(\mathrm{x}<$ 0.06). Thus even in the InGaN/ AlInGaN MQW structures described in this report, the role played by In could be significantly reduced from that of blue and green LED structures with higher In compositions in the InGaN QWs.

In an effort to further elucidate these issues, we have performed a number of photoluminescence studies of InGaN, GaN and AlGaN MQW and buik structures. In particular, we have performed temperature-dependent photoluminescence (PL) spectroscopy measurements on a number of MOVPE grown $\mathrm{In}_{\mathrm{x}} \mathrm{Ga}_{1-\mathrm{x}} \mathrm{N}$ epilayers in the low In composition regime $(\mathrm{x}<0.10)$ [12]. This composition regime was chosen to examine whether a clear trend in optical efficiency and temperature dependent quenching of PL intensity can be found with the addition of just small amounts of In. Our work has also intentionally focused on relatively thick $(0.2 \mu \mathrm{m})$ and doped bulk InGaN epilayers so that the role of piezoelectric field effects would be minimized [13]. This work is therefore distinct from the majority of the previously reported work that has focused on InGaN quantum wells with higher $(x \geq 0.1)$ In composition.

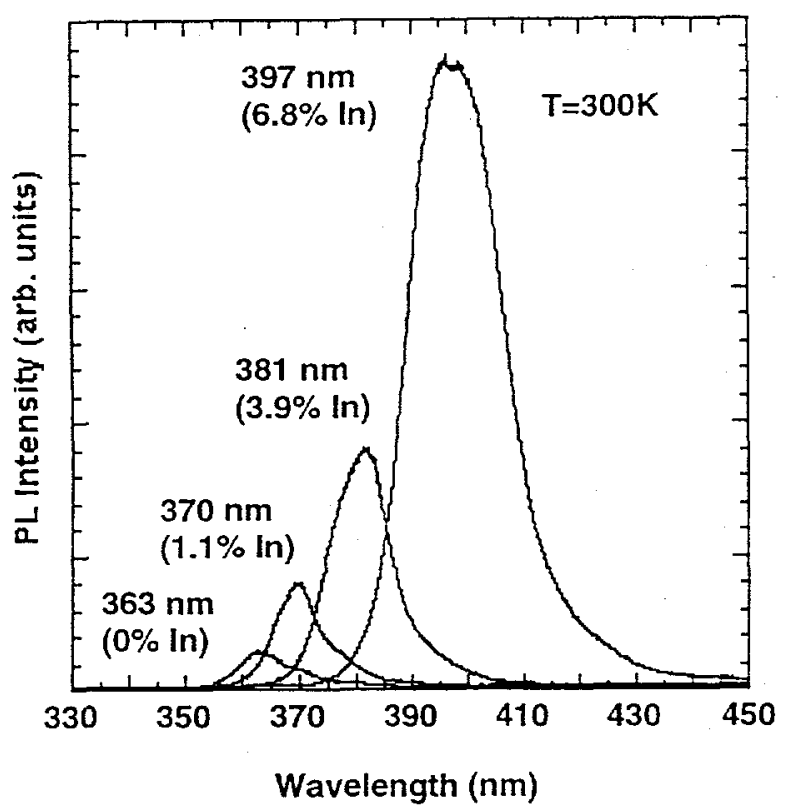

Figure 2a : Room temperature PL spectra of InGaN bulk epilayers [12].

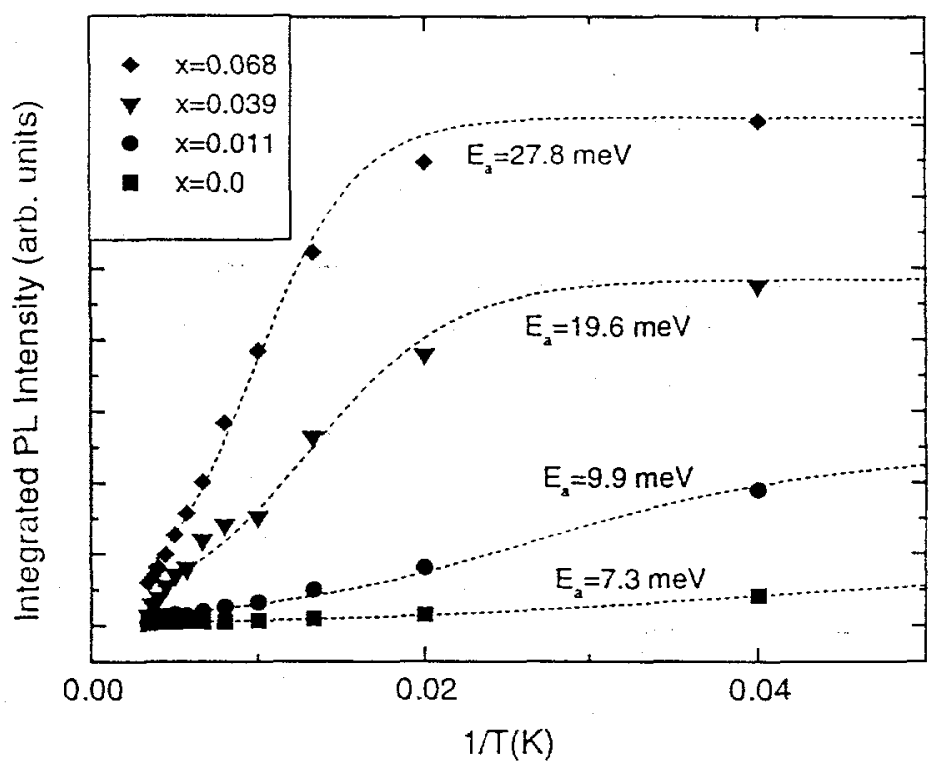

Figure 2b: Temperature dependent integrated PL intensity of InGaN bulk epilayers with In composition $x$. The Ea values are derived from fitting the data to equation 1 [12].

The PL measurements were performed using a HeCd laser $(325 \mathrm{~nm})$ at a low power density of approximately $30 \mathrm{~W} / \mathrm{cm}^{2}$. A 0.3 meter spectrometer with an integrated UV enhanced CCD detector was used, with a spectral resolution of approximately $0.2 \mathrm{~nm}$. The room temperature PL spectra for four InGaN epilayer samples is shown in Figure $2 \mathrm{~b}$. A strong increase in the integrated PL intensity is seen as In composition is increased, with more than a $25 \mathrm{X}$ increase as the peak wavelength shifts from 363-397 $\mathrm{nm}$. The data suggest that the optical efficiency is highly dependent on the In composition. The full temperature dependence of the integrated PL intensity is plotted in an Arrhenius plot in Figure $2 \mathrm{~b}$. The data is fit with the following formula [14]

$$
\mathrm{I}=\mathrm{I}_{0} /\left[1+\alpha \exp \left(-\mathrm{E}_{\mathrm{a}} / \mathrm{kT}\right)\right]
$$


Here the non-radiative decay is assumed to be thermally activated such that the non-radiative lifetime $\tau_{\mathrm{nr}}=\tau_{\mathrm{o}} \exp \left(\mathrm{E}_{\mathrm{a}} / \mathrm{kT}\right)$ and $\mathrm{E}_{\mathrm{a}}$ is the activation energy for PL quenching. The parameter $\alpha$ is equal to $\tau_{\mathrm{r}} / \tau_{\mathrm{o}}$ where $\tau_{\mathrm{r}}$ is the radiative lifetime. From the $E_{\mathrm{a}}$ values, we see a systematic increase in the activation energy as the indium composition is increased. The full temperature dependent data thus give further support of the hypothesis that increasing In composition improves the optical efficiency of the materials. Through the increasing $E_{a}$ values with increasing In composition, we see that the In is reducing the effectiveness of non-radiative recombination mechanisms in quenching the PL intensity. Whether this effect is due to carricr localization or a modification of the nature of the non-radiative centers can not be determined from the data.

Additional temperature dependent PL measurements were performed on InGaN, GaN and AIGaN MQW structures. The InGaN QW structures were supplied by Meijo University and have 10 periods of $23 \AA$ thick $\operatorname{In}_{0.21} G_{0 N} N_{0.79} N$ QWs with GaN barriers. The GaN MQW structure was grown at Sandia National Laboratories and consists of 4 periods of $30 \AA$ thick GaN quantum wells with $\mathrm{Al}_{0.20} \mathrm{Ga}_{0.80} \mathrm{~N}$ barriers. It should be noted that this $\mathrm{GaN} \mathrm{MQW}$ structure was grown on an AlGaN buffer so that the QW emission would not be confused with emission from a thick GaN buffer layer. The AlGaN MQW structure was identical to the GaN MQW structure except that approximately 5\% aluminum was added to the QWs. In Figure 3a, we show the low temperature ( $\mathrm{T}=10 \mathrm{~K}$ ) photoluminescence spectra for the three MQW structures. In Figure $3 \mathrm{~b}$, we show the temperature dependence of the peak PL intensity for the three MQW structures. A strong distinction is seen in the total drop in peak PL intensity. In particular, the InGaN MQW structure experiences a relatively small (4X) loss in peak PL intensity from 10-300K, while the peak PL drop is 60X and 1000X for the GaN MQW and AlGaN MQW structure, respectively. In the quantitative comparison of the PL data for these three MQW structures, it is important to recognize that the growth conditions for the visible and UV MQW structures were quite different, and it is possible that further optimization of the growth conditions for the UV MQW structures would improve the performance. Nevertheless, this data is a good representation of the performance of our current GaN MQWs and high quality InGaN MQWs. In this data, we see evidence

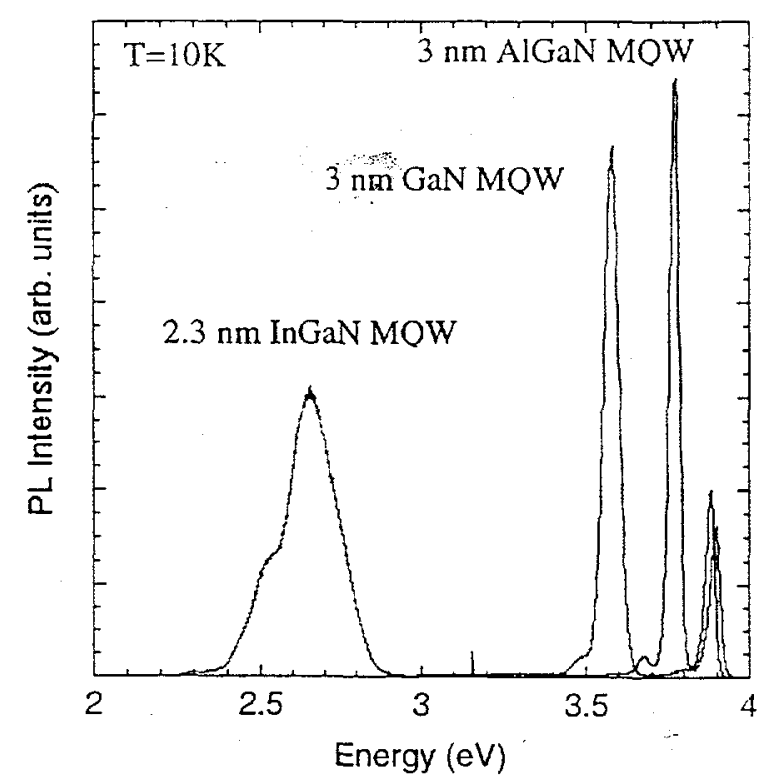

Figure 3a: $T=10 \mathrm{~K}$ PL of InGaN, GaN and AlGaN MQWs.

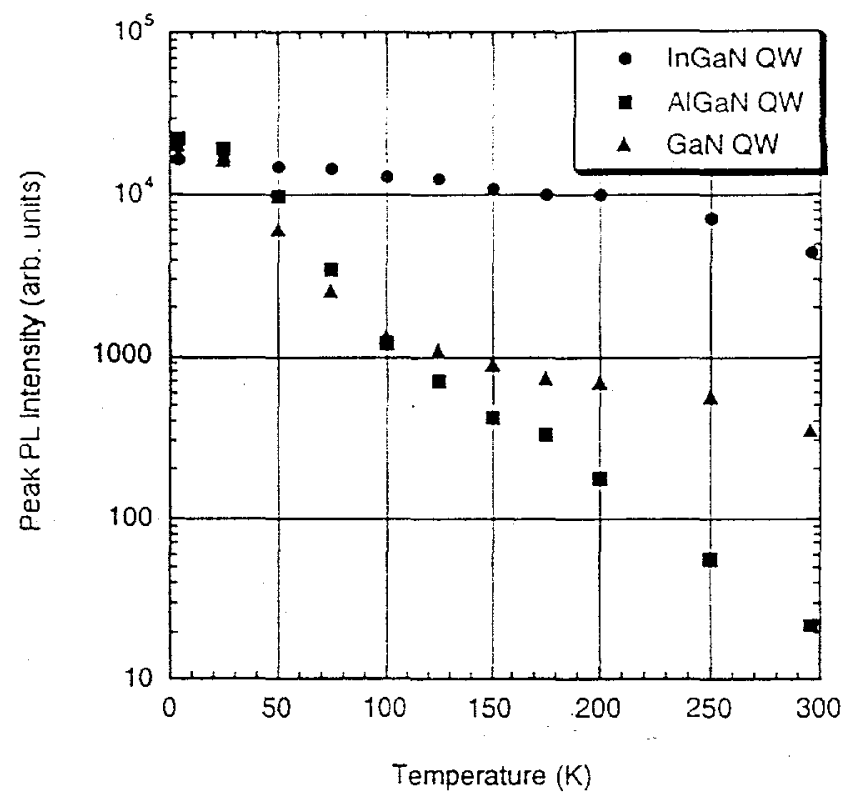

Figure 3b: Temperature dependence of peak PL intensity for InGaN, GaN and AlGaN MQWs.

that the discrepancy in the optical efficiency between the visible and UV MQW structures is highly temperature dependent. In particular, the UV MQW structures are more susceptible to non-radiative recombination processes which serve to quench the PL intensities at room temperature. Furthermore, the severe $1000 \mathrm{X}$ drop in intensity from $10-300 \mathrm{~K}$ for the AlGaN MQWs shows the increasing challenge of obtaining high optical efficiency at wavelengths in the $340 \mathrm{~nm}$ region and shorter.

As a final experiment, we performed temperature dependent PL studies of InGaN MQW structures where the In composition was kept to a low value $(-4 \%)$ to enable room temperature emission at $380 \mathrm{~nm}$ in the UV. These structures are similar to the 
MQW region of the LED heterostructures described in section 2. The particular structures grown for PL studies consisted of 10 periods of $47 \AA$ thick InGaN ( $x=0.04$ ) QWs with AlInGaN barriers. A drop of $\sim 5 \mathrm{X}$ in the integrated PL intensity from $10-300 \mathrm{~K}$ is seen [13], which is similar to the performance of the blue-emitting InGaN MQW structure described in Figure 3.

In summary, the spectroscopic studies that we have performed on InGaN bulk films and InGaN, GaN and AlGaN MQWs have shown that samples with In-containing QWs can have significantly higher optical efficiency than samples with no In in the active region. This result might suggest that GaN/AIGaN MQWs may not be intrinsically capable of performing to the level of InGaN near UV and visible MQWs. To improve the performance of GaN/AIGaN MQWs, a further measure of growing the structures on epitaxially laterally overgrow $\mathrm{GaN}$ to significantly reduce dislocation densities may serve to largely improve the non-radiative recombination problem. Indeed, studies by Mukai et. al. have shown that GaN/AIGaN DH LEDs had up to $2 \mathrm{X}$ increased output powers when this technique was employed [16].

\subsection{Internal Absorption Effects for Shorter Wavelength $(\lambda<370 \mathrm{~nm})$ UV LEDs}

In consideration of the challenges in obtaining high efficiency UV LEDs, one must also seriously consider the detrimental role played by internal absorption for UV LEDs with $\lambda<370 \mathrm{~nm}$. At the heart of this problem is the fact that most nitridebased LEDs rely on 3-4 $\mu \mathrm{m}$ thick $\mathrm{n}$-GaN buffer layers and $\sim 0.1 \mu \mathrm{m}$ thick $\mathrm{p}-\mathrm{GaN}$ capping layers for good current spreading and low contact resistance. While these GaN layers are rather transparent for blue LEDs operating at $450 \mathrm{~nm}$, they become strongly absorptive as the QW emission wavelength reaches $370 \mathrm{~nm}$ and shorter wavelengths. This effect has been described by both Mayer et. al. [17] and Mukai et al. [18] as a significant contribution to the sudden loss of optical efficiency for $\lambda<$ $370 \mathrm{~nm}$.

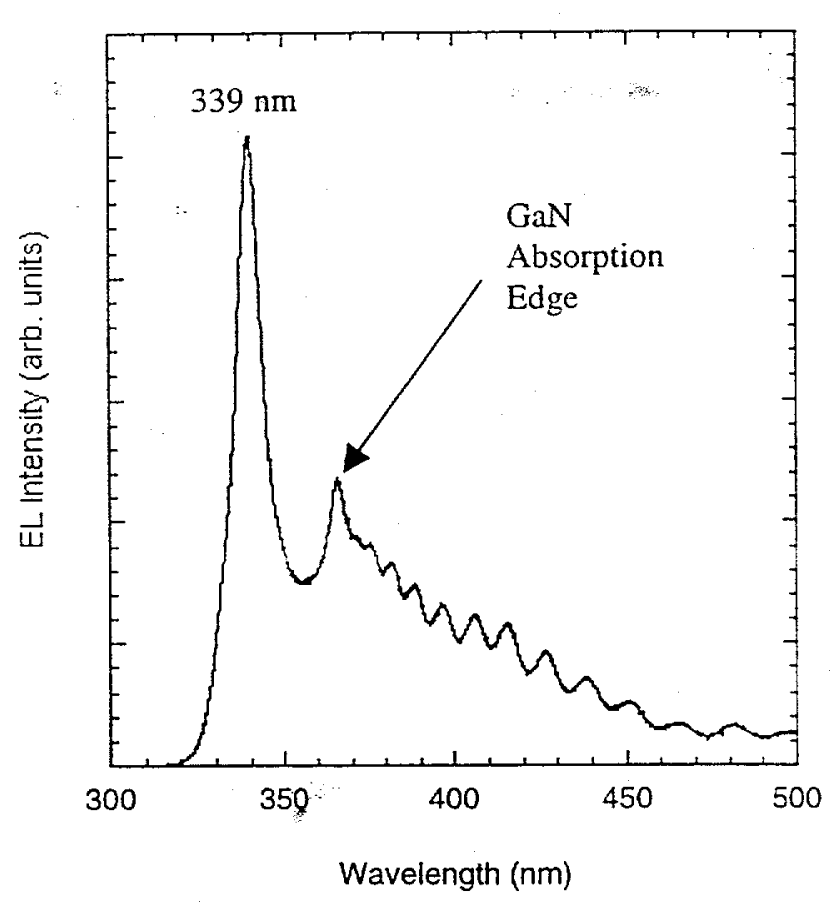

Figure 4: EL spectra for AlGaN MQW LED at $80 \mathrm{~mA}$.
We have most clearly seen this effect in the electroluminescence (EL) spectra of AIGaN MQW LEDs operating at $340 \mathrm{~nm}$. These LEDs have similar AIGaN MQW active regions to those described in section 3.1. In Figure 4, we show the EL spectrum at $80 \mathrm{~mA}$ injected current. A clear delineation of the $\mathrm{GaN}$ absorption edge can be seen at approximately $365 \mathrm{~nm}$. Fabry-Perot (F-P) oscillations exist in the spectrum for wavelengths below this absorption edge, signifying the transparency of the sample at those wavelengths. In contrast, the $340 \mathrm{~nm}$ peak from the AlGaN QWs is significantly reduced in intensity compared to what one would expect from the tail emission and shows no F-P oscillations. Thus, it is clear that if one needs high efficiency at these shorter wavelengths, a more transparent buffer layer, such as one consisting of higher bandgap AlGaN or AlInGaN must be employed. Progress in AlGaN buffer layers has been reported by Takeuchi et. al. [19], who have demonstrated growth of a blue laser structure on an $\mathrm{Al}_{0.03} \mathrm{Ga}_{0.97} \mathrm{~N}$ $/ \mathrm{Al}_{0.06} \mathrm{Ga}_{0.94} \mathrm{~N}$ buffer layer.

\subsection{AlGaN Cladding Designs: Critical thickness Limitations and Carrier Leakage}

Another challenge in the growth and design of nitride-based UV LEDs is the fact that one needs increasingly higher bandgap materials for the barrier and cladding layers. These high bandgap layers are needed to ensure sufficient carrier confinement in the quantum wells, as well as to reduce carrier leakage out of the active region. At present, $\mathrm{Al}_{\mathrm{x}} \mathrm{Ga}_{1-\mathrm{x}} \mathrm{N}$ alloys with $\mathrm{x}=0.10$ 0.20 are most commonly used as cladding layers in InGaN and GaN LEDs. Since AlGaN is tensile strained when grown pseudomorphically on $\mathrm{GaN}$ buffer layers, there is a critical thickness before dislocations and/or cracking sets in. This requirement puts a strong limitation on the thickness and composition that one may use to reduce electron leakage in the 
LEDs. In general, these thickness limitations are much more critical for laser diodes than for LEDs, since one needs a much thicker cladding layer to provide optical confinement. Nevertheless it is important to recognize that even for UV LEDs, one must make a compromise between the cladding composition and thickness and the optimal confinement. As will be discussed in section 5, this problem can be eliminated if one could grow high bandgap AlInGaN alloys lattice matched to $\mathrm{GaN}$ for cladding layers or grow structures on $\mathrm{AlGaN}$ buffer layers. In this section, however, we will focus on the issues of critical thickness and carrier leakage of UV LEDs with AlGaN claddings.

The critical thickness for stress relaxation in AlGaN/GaN heterostructures has been studied by Hearne, et al. [20] through insitu stress measurements during MOVPE growth. This work determined that the observed critical thickness for cracking in AlGaN was consistent with the Griffith equation estimated critical thickness. Using the values from Ref. 20, the approximate critical thickness for an $\mathrm{Al}_{0.3} \mathrm{Ga}_{0.7} \mathrm{~N}$ epilayer on $\mathrm{GaN}$ is $700 \AA$, for an $\mathrm{Al}_{0.2} \mathrm{Ga}_{0.8} \mathrm{~N}$ epilayer on $\mathrm{GaN}$ is $0.15 \mu \mathrm{m}$, and for an $\mathrm{Al}_{0.1} \mathrm{Ga}_{0.9} \mathrm{~N}$ layer on $\mathrm{GaN}$ is $0.6 \mu \mathrm{m}$.

The effects of exceeding the critical thickness can be clearly seen in Figure 5, where we show a top-view photograph of a $100 \mu \mathrm{m}$ diameter mesa-etched GaN MQW LED with $\mathrm{Al}_{0.2} \mathrm{Ga}_{0.8} \mathrm{~N}$ barrier and cladding layers. The total thickness of the AlGaN layers exceeds $0.2 \mu \mathrm{m}$. The cracking networks are especially visible in the etch-exposed $\mathrm{n}$-GaN layer, where preferential etching in the cracks has made them more pronounced. I-V characterization of such LED structures yielded extremely poor reverse leakage characteristics, as shown for a number of cracked devices in Figure $5 \mathrm{~b}$.

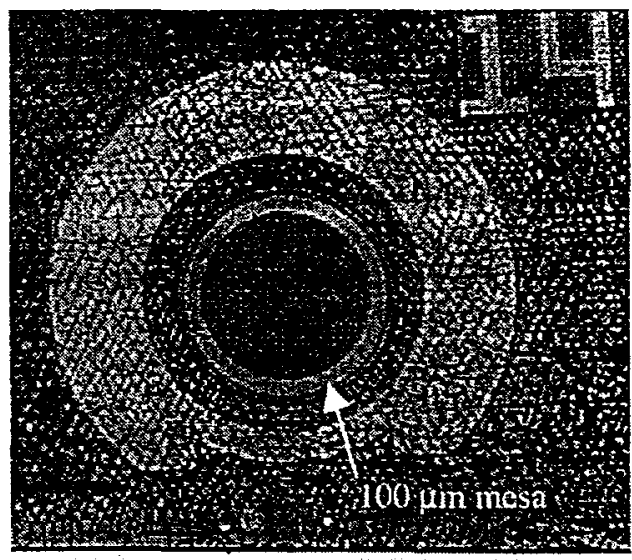

Figure 5a: Top view photograph of a GaN/AlGaN MQW LED with $>0.2 \mu \mathrm{m}$ total thickness of $\mathrm{Al}_{0.2} \mathrm{Ga}_{0.8} \mathrm{~N}$ barrier.

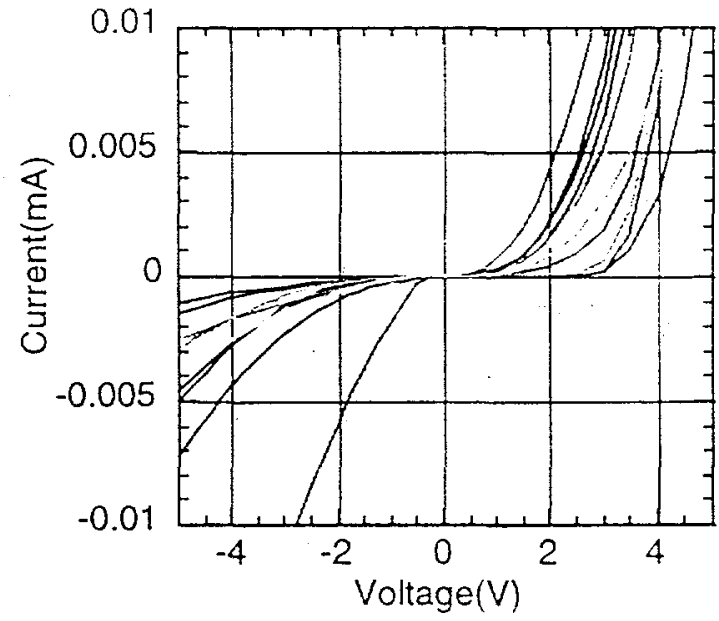

Figure 5b: I-V characteristics for several cracked GaN /AlGaN MQW LEDs

Given these limitations in Al composition and thickness, it is useful to evaluate the expected carrier leakage from GaN MQW structures with various composition AlGaN claddings. We have modeled the carrier leakage in a GaN/AlGaN single QW structure grown along the c-axis of the hexagonal wurtzite crystal structure. This particular structure assumes the AlGaN layers are relaxed and that the GaN QW is compressively strained. Such a structure is relevant for UV LED structures grown on transparent AlGaN buffer layers. The basic approach is detailed in Ref. 21. It involves calculating the bandstructure and carrier distribution in both the confined QW states and the unconfined barrier states as a function of injected carrier density. Piezo-electric and spontaneous polarization effects [22] are included in the calculation. Carrier leakage is determined by assuming that the unconfined states of the QW structure, which contribute to current leakage via carrier recombination, drift or diffusion, may be populated through thermalization with the population in the bound QW states. We determine the carrier leakage by the ratio of the unconfined carrier density to the confined carrier density. A schematic of the GaN SQW heterostructure and the processes described by the model are shown schematically in Figure 6 . We use a $6 \times 6$ LuttingerKohn Hamiltonian and the envelope approximation [23] to compute the hole energy dispersions and the optical dipole matrix elements. Input parameters to the calculation are the bulk wurtzite materials parameters [24] for the binary alloys. A conduction band offset of 0.67 is assumed. 


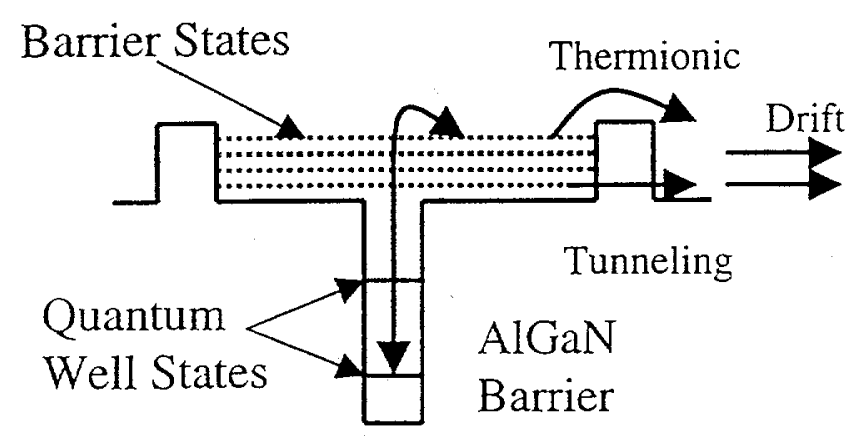

\section{GaN QW}

Figure 6: Schematic of the GaN SQW heterostructure and states involved in the carrier leakage model

We first explore the carrier leakage using a relatively low bandgap $\mathrm{Al}_{0.1} \mathrm{Ga}_{0.9} \mathrm{~N}$ barrier and plot the electron and hole densities in unconfined states versus total carrier population. In Figure 7 we show the results of our calculations for a $2 \mathrm{~nm}$ and a $4 \mathrm{~nm}$ thick GaN QW. The calculations assume $T=300 \mathrm{~K}$.
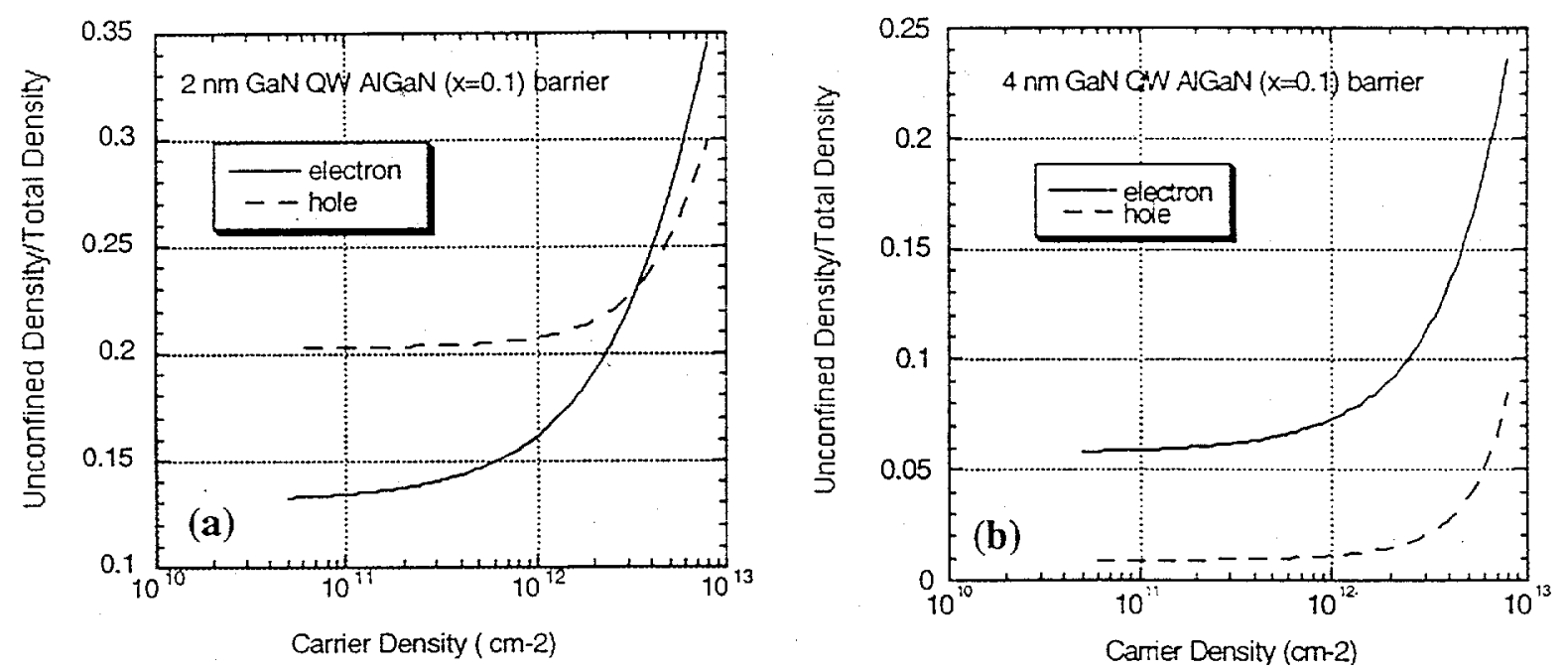

Figure 7: Electron (solid curve) and hole (dashed curve) densities in unconfined states versus total (confined and unconfined) carrier population at $\mathrm{T}=300 \mathrm{~K}$ for (a) $2 \mathrm{~nm} \mathrm{GaN} \mathrm{QW/Al} 0_{0.1} \mathrm{Ga}_{0.9} \mathrm{~N}$. barrier structure and (b) $4 \mathrm{~nm} \mathrm{GaN}$ $\mathrm{QW} / \mathrm{Al}_{0.1} \mathrm{Ga}_{0.9} \mathrm{~N}$ barrier structure.

For both the $2 \mathrm{~nm}$ QW and the $4 \mathrm{~nm} \mathrm{QW}$, we see that the unconfined carrier populations are relatively insensitive to the total carrier density until one reaches total densities of approximately $1 \times 10^{12} \mathrm{~cm}^{-2}$. This density is higher than that typically used for LED operation, and thus the carrier leakage should be relatively insensitive to injected current for LED operation at modest injection levels. For UV laser diodes, however, where one would expect the threshold carrier densities to be as high as $1 \times 10^{13} \mathrm{~cm}^{-2}$, our calculations predict a significant loss of $20-35 \%$ of the carriers due to leakage. For the narrower $2 \mathrm{~nm}$ QW in the low injection regime $\left(<1 \times 10^{12} \mathrm{~cm}^{-2}\right)$, our analysis shows that hole leakage is dominant and can represent a carrier loss of up to $20 \%$. In contrast, electron leakage dominates for the wider $4 \mathrm{~nm}$ well and is reduced to $~ 6 \%$ at these injection levels. The dominance of hole leakage for the $2 \mathrm{~nm}$ well can be explained by the fact that the valence band offset is significantly smaller than the conduction band offset ( $\Delta \mathrm{Ec}=0.67 \mathrm{Eg}$ is assumed) and the confined quantum well states are relatively close to the unconfined levels in this narrow well. In the thicker $4 \mathrm{~nm}$ well, the confined states have lower energy relative to the unconfined states and can provide sufficient confinement for the holes. In this case, the lighter electrons 
experience more leakage. Although the $4 \mathrm{~nm}$ well is clearly superior in terms of reduced leakage, it is important to note that piezoelectric effects, which would significantly reduce electron-hole overlap, are more severe for the thicker wells.

We further explore the importance of higher Al composition claddings by calculating the carrier leakage for $2 \mathrm{~nm}$ GaN QWs with $\mathrm{Al}_{0.1} \mathrm{Ga}_{0.9} \mathrm{~N}$ barriers and $\mathrm{Al}_{0.2} \mathrm{Ga}_{0.8} \mathrm{~N}$ barriers. In Figure 8, we assume an injected carrier density of $1 \times 10^{11} \mathrm{~cm}^{-2}$ and plot the unconfined carrier density as a function of LED temperature. From this calculation, we see that the benefit of the higher bandgap $\mathrm{Al}_{0.2} \mathrm{Ga}_{0.8} \mathrm{~N}$ barrier is quite significant, showing only a $0.5 \%$ electron leakage at $300 \mathrm{~K}$. The stronger confinement of this structure leads to electron leakage being dominant, as for the thicker QW case in Figure $7 \mathrm{~b}$. The elcctron and hole leakage levels increase strongly with temperature, resulting in up to $2.7 \%$ electron leakage at $100^{\circ} \mathrm{C}$ above room temperature.
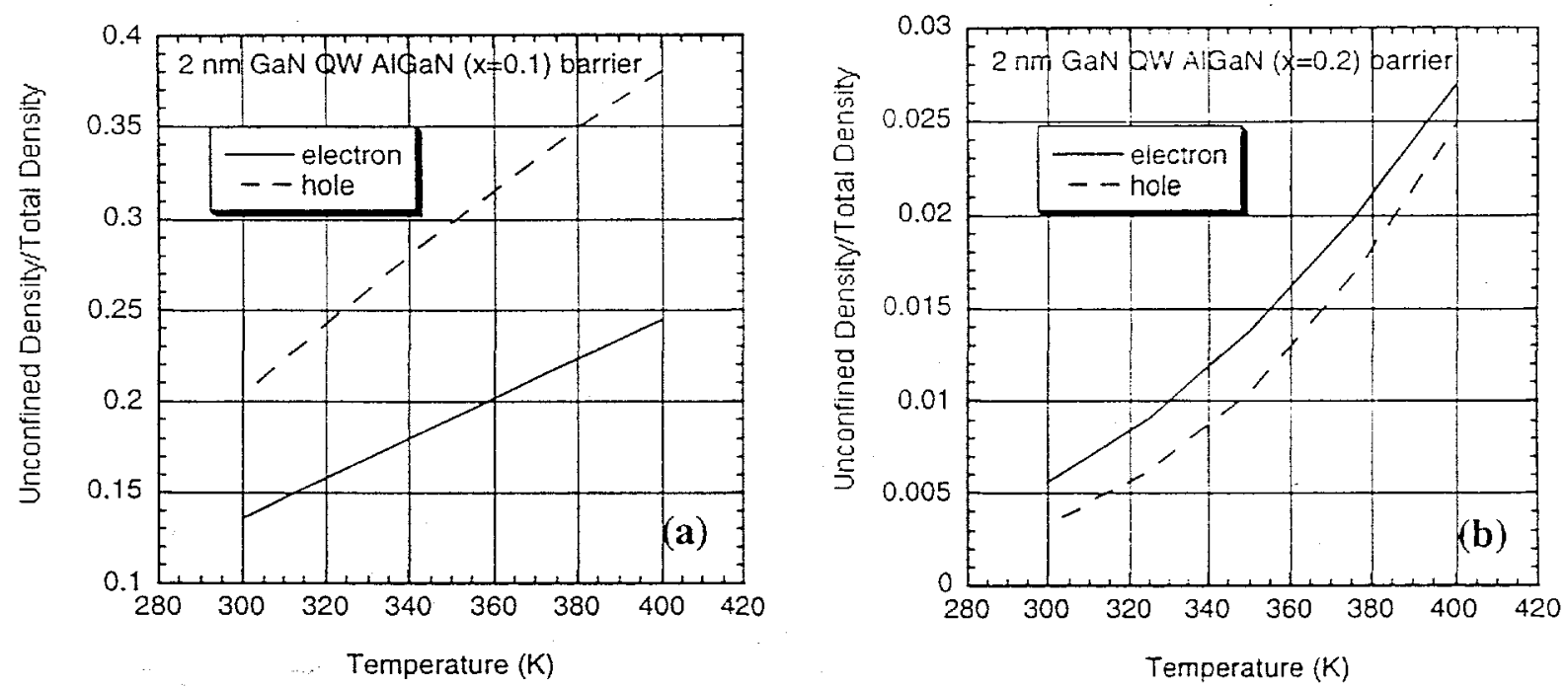

Figure 8: Electron (solid curve) and hole (dashed curve) densities in unconfined states versus total (confined and unconfined) carrier population as a function of temperature for (a) $2 \mathrm{~nm} \mathrm{GaN} \mathrm{QW/A} \mathrm{l}_{0.1} \mathrm{Ga}_{9.9} \mathrm{~N}$ barrier structure and (b) $2 \mathrm{~nm}$ $\mathrm{GaN} \mathrm{QW} / \mathrm{Al}_{0.2} \mathrm{Ga}_{0.8} \mathrm{~N}$ barrier structure. A total carrier concentration of $1 \times 10^{11} \mathrm{~cm}^{-2}$ was assumed.

In summary, we have shown that carrier leakage can significantly reduce the efficiency of GaN/AIGaN MQW LEDs. A barrier $\mathrm{Al}$ composition of $\mathrm{x}=0.2$ is sufficient to reduce carrier leakage to $<1 \%$ of the total carrier population for $2 \mathrm{~nm}$ thick $\mathrm{GaN} \mathrm{QWs}$ at $300 \mathrm{~K}$. In-situ stress measurements suggest that up to $0.15 \mu \mathrm{m}$ of $\mathrm{Al}_{0.2} \mathrm{Ga}_{0.8} \mathrm{~N}$ barrier and cladding layers can be employed before large scale cracking sets in. For shorter wavelength UV LEDs (<350 nm), such as those employing AlGaN QWs, issues of carrier confinement and critical thickness are more critical.

\section{PERFORMANCE OF GaN/AIGaN MQW LEDS for $\lambda<360 \mathrm{~nm}$}

We have fabricated GaN/AlGaN MQW LEDs using the heterostructure design shown described in section 2. $100 \mu \mathrm{m}-250$ $\mu \mathrm{m}$ mesas were defined by inductively coupled plasma (ICP) etching. Ti/Al/Ti/Au was used as the $\mathrm{n}$-contact, and Ni/Au oxidized to form semi-transparent $\mathrm{NiO}[25]$ was used as the p-contact. Initial structures had a relatively narrow $30 \AA \mathrm{QW}$, and electroluminescence peaked at $354 \mathrm{~nm}$ [3], as shown in Figure 9a. Th L-I-V data shown in Figure 9b was taken with a calibrated Si detector in close proximity to the sample, and demonstrates $12 \mu \mathrm{W}$ of output power at a current of $20 \mathrm{~mA}$ ( $180 \mathrm{~A} / \mathrm{cm}^{2}$ for these devices), and a turn-on voltage of approximately $4 \mathrm{~V}$. This performance results in an external quantum efficiency of below $0.1 \%$, which is largely due to the internal absorption effects. The EL FWHM of $5.8 \mathrm{~nm}$ is significantly narrower than that reported for InGaN blue and green LEDs; a feature which is often desirable for spectroscopic applications. 

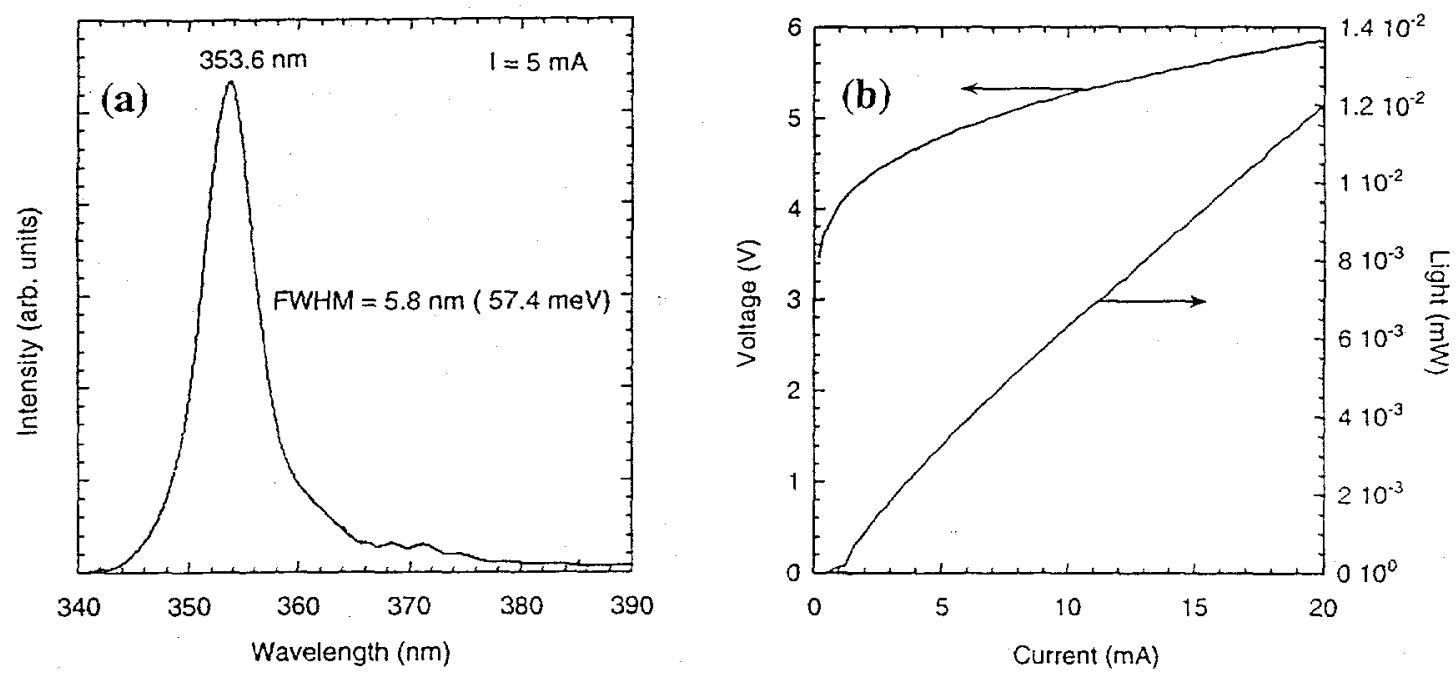

Figure 9: (a) Electroluminescence spectrum of $30 \AA$ GaN/AlGaN MQW LED at $5 \mathrm{~mA}$. (b) Light output-current-voltage characteristic for this $120 \mu \mathrm{m}$ diameter mesa-etched device. [3]

We have further optimized the growth of the GaN QW regions as well as explored QW structures emitting at slightly longer wavelengths where the internal absorption effects would be reduced. In Figure 10, we show the performance of a GaN/AIGaN MQW LED with emission at $357.5 \mathrm{~nm}$. From the EL spectrum, and one can see that the tail of the spectrum is enhanced due to the strongly reduced absorption of the GaN buffer layer at those wavelengths. The L-I data shown in Figure $10 \mathrm{~b}$ was taken from LEDs bonded to TO-headers (no encapsulation or lens), and using an integrating sphere coupled to a calibrated Si detector. These LEDs showed $>100 \mu \mathrm{W}$ output at currents up to $50 \mathrm{~mA}\left(\sim 100 \mathrm{~A} / \mathrm{cm}^{2}\right.$ for these larger devices). While these powers are at least an order of magnitude less than that measured from commercially available blue and green LEDs, such powers are already sufficient for a number of fluorescence-based sensing applications.
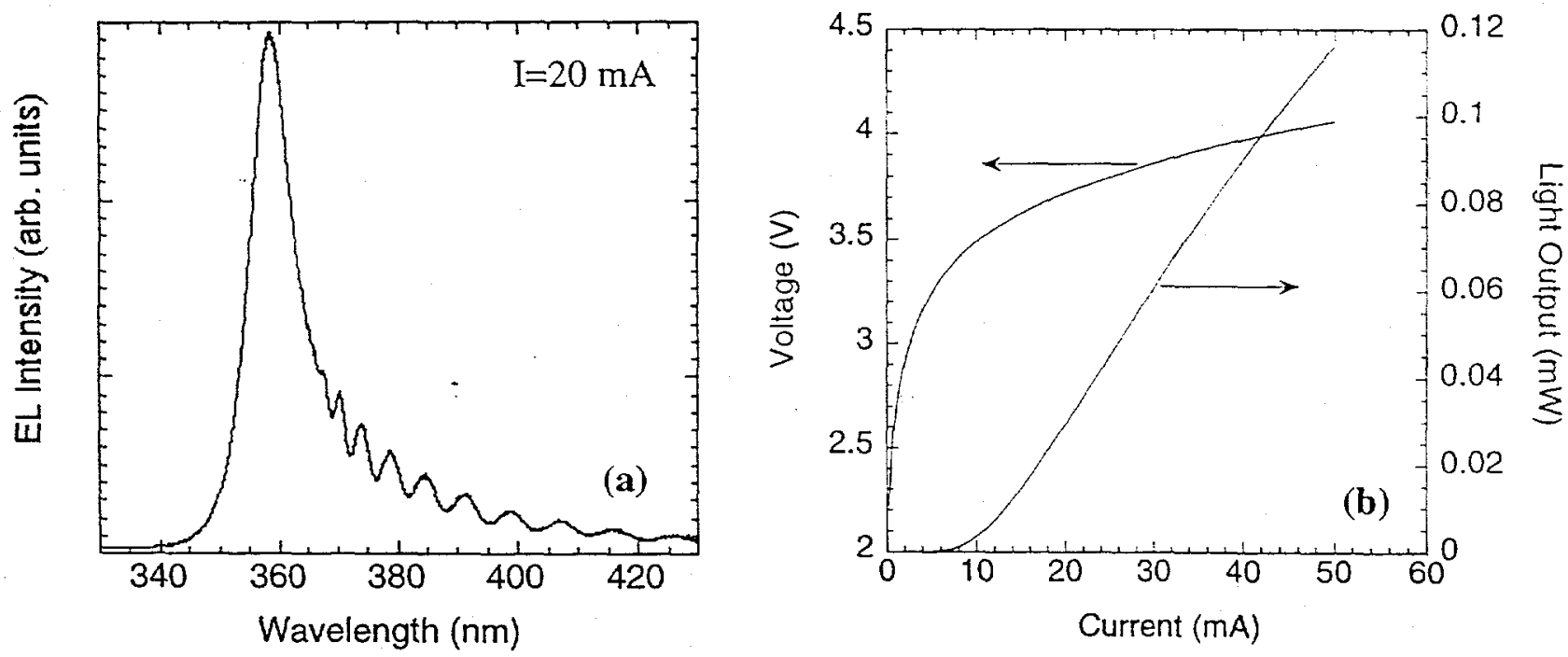

Figure 10: (a) Electroluminescence spectrum of a GaN/AIGaN MQW LED at $20 \mathrm{~mA}$. (b) Light output-current-voltage data for this larger device. 


\section{PERFORMANCE OF InGaN/AIInGaN LEDS FOR $370 \mathrm{~nm}<\lambda<390 \mathrm{~nm}$}

We have further explored UV LEDs employing AlInGaN quaternary alloys in the active region. These materials offer a great deal of flexibility in that they can be lattice matched to GaN and AlGaN buffer layers and thus critical thickness and cracking issues may be eliminated. Despite the obvious benefits of these quaternary alloys, the materials growth is quite challenging due partly to very dissimilar optimal temperatures for $\mathrm{Al}$ and In incorporation. As a result, very few reports have been made on the growth and optical properties of these materials [26,27]. The buffer and cladding layer designs are similar to GaN/AlGaN MQW structures, but the active region consists of $47 \AA$ thick $\operatorname{In}_{x} \mathrm{Ga}_{1-x} \mathrm{~N}$ quantum wells with $x=0.04$ and $48 \AA$ thick $\mathrm{Al}_{\mathrm{y}} \mathrm{In}_{\mathrm{x}} \mathrm{Ga}_{1-\mathrm{x}-\mathrm{y}} \mathrm{N}$ barriers with $\mathrm{x}=0.04$ and $\mathrm{y}=0.14$. This quaternary alloy composition has been found to have a room temperature PL peak at $-357 \mathrm{~nm}$. As described in section 3.1, we have found that active regions consisting of this quantum well structure have very good optical efficiency.

In Figure 11, we show the electroluminescence spectrum and L-I-V data for a InGaN/AlInGaN MQW LED. The EL spectrum, taken at $20 \mathrm{~mA}$, shows a peak at approximately $386 \mathrm{~nm}$ and a FWHM of $10 \mathrm{~nm}(84 \mathrm{meV})$. The Fabry-Pcrot oscillations seen throughout the spectrum indicate that these longer wavelengths experience relatively little absorption from the GaN layers in the structure. The L-I-V data, taken on devices bonded to a TO-header and using an integrating sphere and calibrated Si detector, show $0.5 \mathrm{~mW}$ at $50 \mathrm{~mA}\left(\sim 100 \mathrm{~A} / \mathrm{cm}^{2}\right)$ and greater than $1 \mathrm{~mW}$ powers at current levels of $100 \mathrm{~mA}(\sim$ $200 \mathrm{~A} / \mathrm{cm}^{2}$ ). The peak external quantum efficiency of these devices is approximately $0.3 \%$. Work is in progress to optimize growth and design of these structures to achieve higher operating efficiencies.
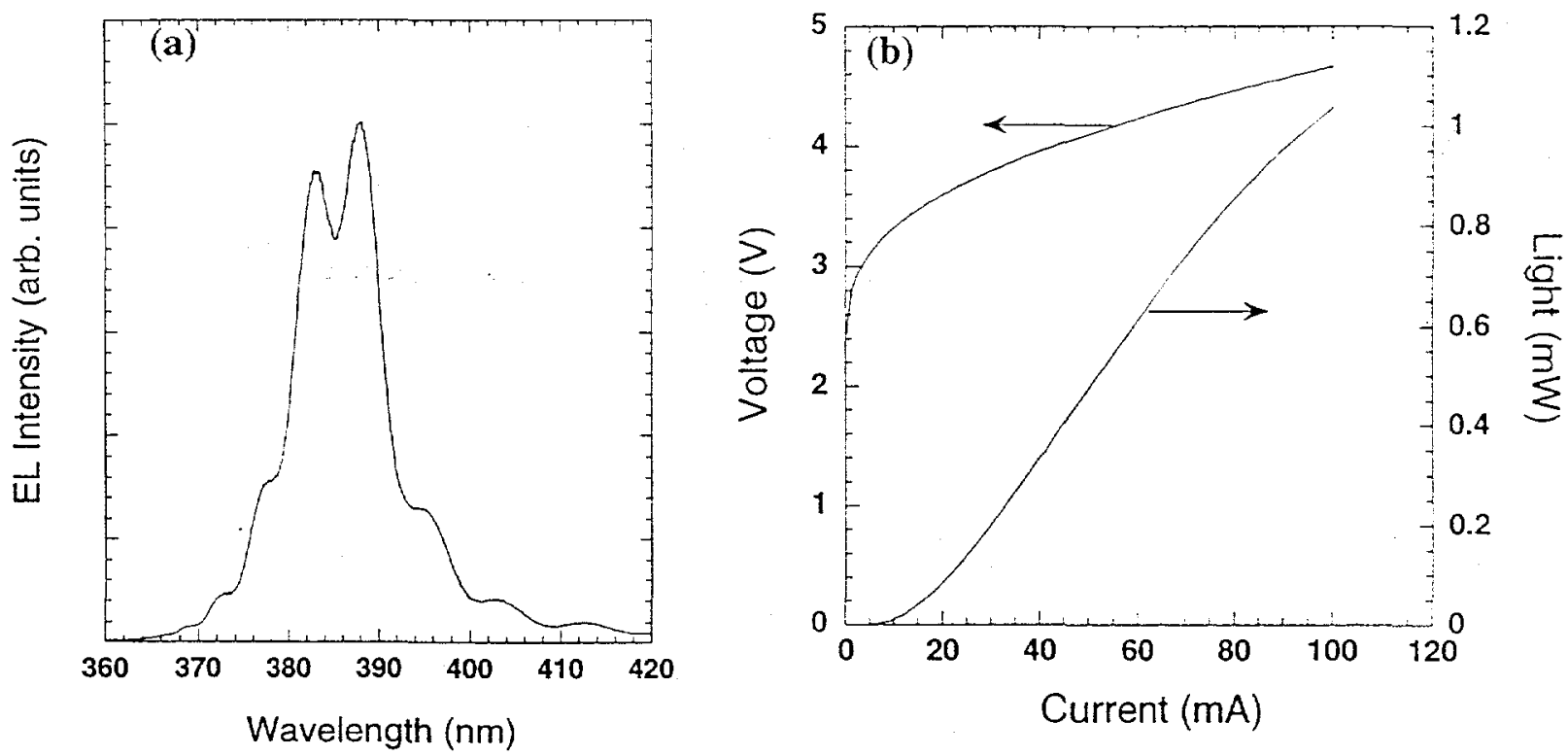

Figure 11: (a) Electroluminescence spectrum of InGaN/AIInGaN MQW LED at $20 \mathrm{~mA}$. (b) Light output-current-voltage data for this device.

\section{SUMMARY}

We have overviewed a number of critical issues that must be addressed to achieve high efficiency UV LEDs. The issue of optical efficiency of UV active regions was explored through photoluminescence studies of InGaN, GaN and AlGaN bulk epilayers and MQW structures. Our results suggest that improved optical efficiency is achieved for active regions with Incontaining alloys in the QWs. Through a carrier leakage model that determined the percentage of carriers in unconfined states of a GaN SQW structure, we deterrinined that $\mathrm{Al}_{0.2} \mathrm{Ga}_{0.8} \mathrm{~N}$ claddings provide relatively good carrier confinement for 2 $\mathrm{nm}$ GaN QW structures. We presented performance data for GaN/AlGaN MQW LEDs with emission in the 354-358 $\mathrm{nm}$ region. Output powers $>100 \mu \mathrm{W}$ have been achieved, which is sufficient for a number of fluorescence-based sensing applications. A new InGaN/AlInGaN MQW UV LED was described which demonstrated $>1 \mathrm{~mW}$ output powers at an emission wavelength of $386 \mathrm{~nm}$. Further optimization of this structure may provide a high efficiency near-UV source suitable for phosphor excitation and white light generation. 


\section{ACKNOWLEDGEMENTS}

The authors acknowledge stimulating technical discussions with S. R. Lee, S. J. Hearne, I. J. Fritz, T. M. Mayer, R. M. Biefeld, and $\mathrm{H}$. Amano. InGaN MQW samples were provided by Prof. $\mathrm{H}$. Amano at Meijo University. Technical contributions by A. V. Nurmikko, Y. K. Song, H. Zhou, N. A. Missert, G. A. Petersen and S. M. Myers are gratefully acknowledged. Sandia is a multiprogram laboratory, operated by Sandia Corporation, a Lockheed Martin company, for the U. S. Department of Energy, under Contract No. DE-AC04-94AL85000.

\section{REFERENCES}

[1] I. Akasaki and H. Amano, Jpn. J. Appl. Phys., 36, 5395( 1997).

[2] T. Mukai, D. Morita and S. Nakamura, J. Cryst. Growth, 189/190, 778 (1998).

[3] J. Han, M. H. Crawford, R. J. Shul, J. J. Figiel, M. Banas, L. Zhang, Y. K. Song, H. Zhou and A. V. Nurnikko Appl. Phys. Lett., 73, 1688 (1998).

[4] J. Han, T. B-. Ng, R. M. Biefeld, M. H. Crawford, and D. M. Follstaedt, Appl. Phys. Lett. 71, 3114 (1997)

[5] J. Han, J. J. Figiel, G. A. Petersen, S. M. Myers, M. H. Crawtord, and M. A. Banas, to appear in Jpn. J. Appl. Phys. (2000)

[6] E. S. Jeon, V. Kozlov, Y.-K. Song, A. Vertikov, M. Kuball, A. V. Nurmikko, H. Liu, C. Chen, R. S. Kern, C. P. Kuo and M. G. Craford, Appl. Phys. Lett., 69, 4194 (1996).

[7] S. Chichibu, T. Azuhata, T. Sota and S. Nakamura, Appl. Phys. Lett., 70, 2822 (1997).

[8] T. Wang, D. Nakagawa, M. Lachab, T. Sugahara, S. Sakai, Appl. Phys. Lett., 74, 3128 (1999) and references therein.

[9] S.F.Chichibu, A.Shikanai, T.Deguchi, A.Setoguchi, R.Nakai, H.Nakanishi, K.Wada, S.P.DenBaars, T.Sota, and S.Nakamura, Jpn. J. Appl. Phys. 39, Part I (2000) (to be published)

[10] Y. Narukawa, S. Saijou, Y. Kawakami, S. Fugita, t. Mukai, S. Nakamura, Appl. Phys. Lett., 74, 558 (1999).

[11] H. Kumano, K. Hoshi, S. Tanaka, I. Suemune, X. Q. Shen, P. Riblet, P. Ramvall and Y. Aoyagi, Appl. Phys. Lett., 75. 2879 (1999).

[12] M. H. Crawford, J. Han, M. A. Banas, S. M. Myers, G. A. Petersen and J. J. Figiel, to appear in MRS Internet J. of Nit. Semicond. Research (2000).

[13] V. Fiorentini, F. Bernadini, Phys. Rev. B, 60, 8849 (1999).

[14] M. Leroux, N. Grandjean, B. Beaumont, G. Nataf, F. Semond, J. Massies, P. Gibart, J. Appl. Phys., 86, 3721 (1999).

[15] M. H. Crawford, unpublished.

[16] T. Mukai and S. Nakamura, Jpn. J. Appl. Phys., 38, 5735 (1999).

[17] M. Mayer, A. Pelzmann, C. Kirchner, M. Schauler, F. Eberhard, M. Kamp, P. Unger and K. J. Ebeling, J. Cryst. Growth, 189/190, 782 (1998).

[18] T. Mukai, M. Yamada and S. Nakamura, Jpn. J. Appl. Phys.,37, L1358 (1998).

[19] T. Takeuchi, T. Detchprohm, M. Iwaya, N. Hayashi, K. Isomura, K. Kimura, M. Yamaguchi, H. Amano, I. Akasaki, Yw. Keneko, R. Shioda, S. Watanabe, T. Hidaka, Y. Yamaoka, Ys. Kaneko and N. Yamada, Appl. Phys. Lett., 75, 2960 (1999).

[20] S. J. Hearne, J. Han, S. R. Lee, J. A. Floro, D. M. Follstaedt, E. Chason and I. S. T. Tsong, Appl. Phys. Lett. (to be published)

[21] W. W. Chow, M. Hagerott Crawford, A. Girndt and S. W. Koch, IEEE J. Sel. Topics in Quant. Electron., 4, 514 (1998). [22] O. Ambacher, J. Smart, J. R. Shealy, N. G. Weimann, K. Chu, M. Murphy, W. J. Schaff, L. F. Eastman, R. Dimitrov, L.

Wittmer, M. Stutzmann, W. Rieger and J. Hilsenbeck, J. Appi. Phys., 85, 3222 (1999).

[23] S. L. Chuang and C. S. Chang, Phys. Rev. B, 54, 2491 (1996).

[24] W. W. Chow and S. W. Koch, Semiconductor-Laser Fundamentals, Springer-Verlag, Berlin, 1999, p. 189.

[25 Jin-Kuo Ho; Charng-Shyang Jong; Chiu, C.C.; Chao-Nien Huang; Kwang-Kuo Shih; Li-Chien Chen; Fu-Rong Chen; JiJung Kai, J. Appl. Phys., 86, 4491 (1999).

[26] M. E. Aurner, S. F. LeBoeuf, F. G. McIntosh and S. M. Bedair, Appl. Phys. Lett., 75, 3315 (1999).

[27] J. Han, J. J. Figiel, G. A. Petersen, S. M. Myers, M. H. Crawford, M. A. Banas and S. J. Hearne, to appear in MRS

Internet J. Nitride Semicond. Research (2000). 